\title{
A New Hybrid Heating System for Solar Cell Module Laminator
}

\author{
Shi Lei ${ }^{\mathrm{a}}$, Chen Li-dong ${ }^{\mathrm{b}}$, Zhang Liang ${ }^{\mathrm{c}}$, Ma Shuying \\ College of Mechanical and Electronic Engineering,Hebei Normal University of Science and \\ Technology, Qinhuangdao,066600,China \\ axnshilei@126.com, ${ }^{\mathrm{b}}$ chentian-940308@163.com, ${ }^{\mathrm{c}}$ Izhang7608@163.com
}

Keywords: solar cell, laminator, hybrid heating system, intelligent temperature control

\begin{abstract}
Solar cell module laminator is important equipment in the production of solar cell, which can press several layers of the glass, EVA plastic, semiconductor wafer and etc together. The heating temperature of laminator is the key parameter to guarantee the quality of solar modules. To improve the heating control precision and decrease the temperature heterogeneity of the table surface, a kind of hybrid temperature-control heating system of solar cell module laminator is introduced. The system consists of the oil-electron hybrid heater, the oil temperature automatic measurement and control system, the temperature setting and display system and other components. The principle of the system is to heat the work table by the electric heater and uses the oil as a heat conduction medium for the temperature compensation, which can make the heating uniformity control precision reach
\end{abstract} $1.2{ }^{\circ} \mathrm{C}$.

\section{Introduction}

Heating system is an important component of solar cell-module laminator, and its performance directly affects the quality of laminated components. At present, the heating system mainly uses two kinds of heating mode. One is the carbon heating plate, another is oil heating system. Carbon heating board heating system mainly uses the carbon heating plate which is set under the surface of the worktable for heating. Because heat transfer between heating plate and working platform is heat transfer between two solids, the effect of heat transfer is influenced by the two bodies contact [1], so it is not easy to control the surface temperature uniformity, temperature gradient is large and uneven, and the heater is likely damaged and working platform is deformed, thereby this affects the quality of laminated components. But oil heating mainly uses heat conducting oil or other media on the workbench for indirect heating, the effect of heat transfer is better, but the heating time is longer, hot oil pipeline is complex [2], and the process of conducting oil flows in a certain way with temperature gradient, the temperature difference of the workbench is larger, the overall temperature uniformity is poor. In addition, solar cell module laminator lift adopts hydraulic system to complete, this makes the system larger, bulky, and difficult to maintain.

In the paper, a new heater system of solar cell-module laminator is discussed, and it is characterized by mixed-temperature control. Heating cores is installed in the heating pipes, and heat conducting oil is used as a heat conducting medium to heat laminated components. Heat conducting oil which flows in a certain way can avoid a large temperature difference on workbench surface, while the temperature measurement and control instrument are adopted to monitor and control oil temperature, so uniformity and stability of the workbench surface working temperature is improved.

\section{Overall structure of the hybrid heating system}

Basic structure of the hybrid heating system. Hybrid heating system consists of the S-shaped pipe in the workbench, electric heating system, and temperature detecting system. The principle is shown in Figure 1.

Working principle. The heating core directly heats the oil in the S-shaped pipe, so the heating efficiency can be greatly improved. The two ends of the S-shaped pipe in workbench are connected to the outlet and the inlet of the pump. The pump can enable the oil which is heated by heating cores to 
circularly flow in the pipe, thus temperature is compensated. Temperature detection and control system includes temperature sensors, signal amplifiers, intelligent temperature control instruments, and solid-state relays etc. Temperature setting and display system is composed of the touch screen. When the solar laminator begins to work, first the power is turned on and oil pump motor begins to supply oil. When the oil flows into the S-shaped pipe, the heating system is started and begins to heat oil; the sensor signal is amplified and passes oil temperature to measurement and control instrument. Temperature measurement and control instrument completes signal analysis, when the measured temperature and working temperature have deviations, the information will be sent to its internal single-chip microcomputer by the temperature measurement and control instrument, and the single-chip microcomputer through programs controls solid state relay on/off, then the temperature of heating cores is adjusted. At the same time, temperature measurement and control instrument transfers the signal to the total single-chip microcomputer through the MAX485 chip interface, and the touch screen displays the measured temperature.

\section{Design of key components}

Structure design of the heating part. Heating part is made up of the S-shaped pipe and internal electric heating cores. The oil pump circulates oil round the S-shaped pipe. Electric heating cores heat wire components set in the heating body, and which are installed in the S-shaped pipe. One end of the pipe connects with the pipe connector, and the other end connects with the temperature measurement and control instrument through the chip interface. A heating unit consists of three cores component, and temperature measurement and control instrument controls the solid state relay on/off, so as to control the heating cores. Thus, using the heating cores to heat the S-shaped pipe and hot oil in the pipe to compensate temperature, the problem of temperature gradient and heating uniformity are improved effectively. The structure is shown in Figure 1.

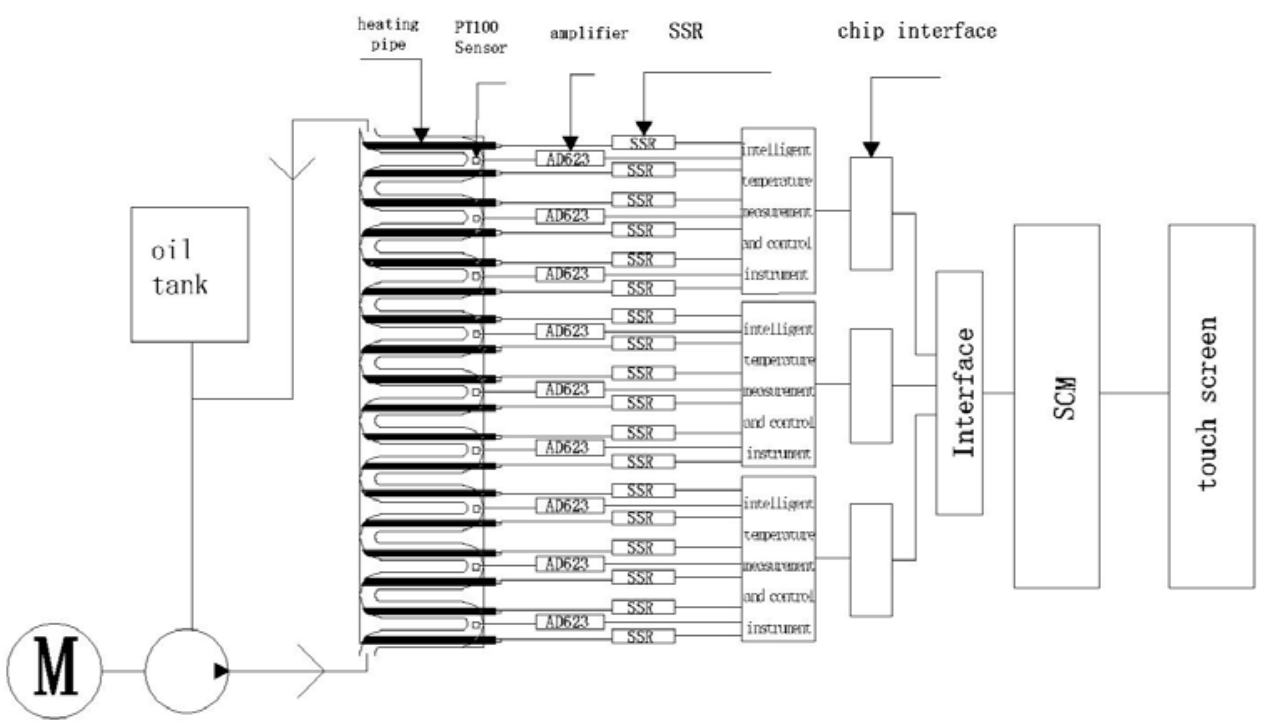

Figure 1 The structure of the mixed heating system

Temperature measurement and control system. Temperature measurement and control system consists of sensors, signal amplifiers, temperature measurement and control instruments and solid state relays. The composition and working principle are shown in Figure 2. PT100 platinum resistance is used as a sensor to measure the temperature, and converts the temperature signal to electrical signal, the signal is amplified through AD623 amplifier and is input temperature measurement and control instrument. The signal is compared with the setting value through the internal MCU with expert PID algorithm, and then commands from the MCU are issued to control solid state relay for adjusting heating of the heating cores. 
Hardware system design. Detection and control system hardware consists of PT100 platinum resistance as a temperature sensor, AD623 signal amplifier, intelligent temperature controller that internal MCU chip is STC12C5A32AD and solid state relay. Its structure is shown in Figure 3.

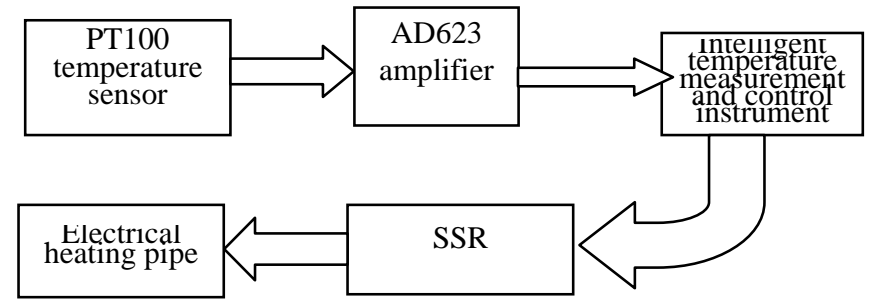

Fig.2 Temperature detection and controlling diagram

a) PT100 platinum resistance temperature sensor.

PT100 platinum resistance temperature sensor utilizes the characteristic of platinum which resistance value changes with temperature changes to measure temperature, The sensor has high accuracy and stability, and wide temperature range which is $-200-650^{\circ} \mathrm{C}$. It has many advantages: a) PT100 platinum resistance temperature sensor has adopted three-wire connection, so that it has a very high accuracy and minimal measurement error. b) The linear of platinum resistance characteristics is good, when the measured temperature range between $-50^{\circ} \mathrm{C}$ and $200^{\circ} \mathrm{C}$, the relationship between temperature and resistance values is close to linear relationship, and the deviation is extremely small, else the electrical performance is stable. c) The platinum resistance is small, resistant to vibration, and with high reliability. d) The platinum resistance has characteristic of high accuracy, high pressure resistance, good stability, long life, easy to be used and low cost are advantages of the platinum resistance [3].

In the process of solar cell lamination, the workbench of solar cell module laminator are required to have high temperature uniformity and stability, and working temperature range is between $20^{\circ} \mathrm{C}$ and $150^{\circ} \mathrm{C}$. In this range, the relationship between temperature and resistance values of platinum resistance is close to linear, and platinum resistance has high accuracy and stability [4], so platinum resistance is suitable for temperature sensor.

b) AD623 amplifier

AD623 is an integrated single power instrument amplifier, it can provide full output in single power supply $(+3 \mathrm{~V}$ to $+12 \mathrm{~V})$. It allows the use of a single gain setting resistor for gain programming to get a better flexibility and accords with 8-pin industry-standard configuration. In the absence of external resistance, AD623 is set to a single gain $(G=1)$. After connected outside resistance, AD623 can set gain by programming, the gain is up to 1000 times. AD623 maintains a smaller error by providing common-mode rejection ratio, and it has low power consumption, wide supply voltage range, full power output and high stability. The amplifier is set betwen the sensor and the MCU.

Intelligent temperature control instrument. Intelligent temperature control instrument can control the working temperature deviation of the heating pipes to a small range with certain accuracy, and then temperature control instrument makes up for the lack of heat and excess heat to keep the temperature of the workbench stable. The temperature control instrument uses the STC12C5A32AD MCU chip, and program uses the expert PID control algorithm. When the measured temperature value collected by thermal resistance deviates from the given value, the MCU can accord to the deviation of the measured signal and gives value to operate the PID control algorithm, so as to control the relay on/off ratio and urges the measured value to restore to the given value, and the purpose of automatic control is achieved.

Intelligent temperature control instrument has a variety of specifications, such as $0-50^{\circ} \mathrm{C}$, $0-100^{\circ} \mathrm{C}, 0-200^{\circ} \mathrm{C}, 0-300^{\circ} \mathrm{C}$ and so on. According to the requirement of working temperature, the design requires the temperature range is $30-150^{\circ} \mathrm{C}$, so the temperature control instrument specification is $0-200^{\circ} \mathrm{C}$ is used, the resolution is $0.1^{\circ} \mathrm{C}$, and the error less than $0.05 \%$ (including sensor error). Backlash can be set to the range which is $0.1 \%$ to $2 \%$; measurement period is $1 / \mathrm{s}$, and it can reflect the external temperature changes. Intelligent temperature control instrument adopts unique 
A/D converter circuit which is controlled by the STC12C5A32AD MCU. It has many advantage characteristics like high resolution, low cost and so on, and it uses digital filter and many other software technologies to improve the accuracy of the instrument.

Solid state relay. $80 \mathrm{Z}$ solid state relay (SSR) is adopted. The SSR is a new non-contact switch device which consists of all electronic components, and it has many advantages such as high reliability, long life, low noise, switching speed fast, strong capability of anti-interference, resistance vibration, resistance impact, damp proof, moisture proof, anti-corrosion, and it is compatible with TTL, CMOS logic circuits, and else it has an unique characteristic of zero conduction [6], so it has good application to control the heating wire.

Software system design. Software program of the temperature control system uses the fuzzy PID control algorithm, and the MCU uses STC89C54RD+ chip to set up temperature and output the temperature value to the touch screen.

First the touch screen transmits the set temperature values to the main MCU, then to the slave MCU, heating system begins to work, the temperature value which is collected by PT100 platinum resistance is transmitted to the slave MCU to start PID calculation and control the heating system. At the same time, the slave MCU transmits the selected temperature to the main MCU and the temperature is displayed on the screen.

Besides the strict and correct logic control, fast and stable high-precision control to temperature is needed, temperature control is required to have a good dynamic and static quality. So, the expert PID controls algorithm used, and main program process as shown in Figure 4.

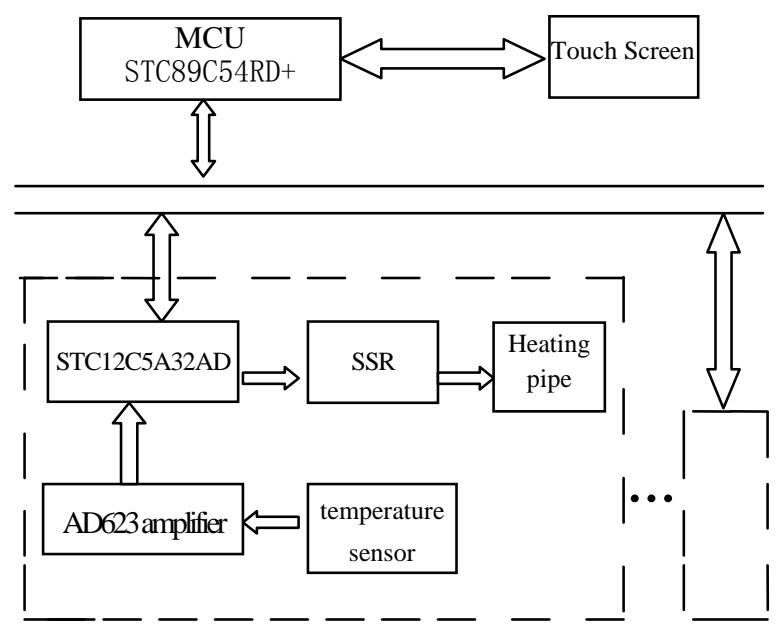

Fig.3 Hardware structure principle diagram

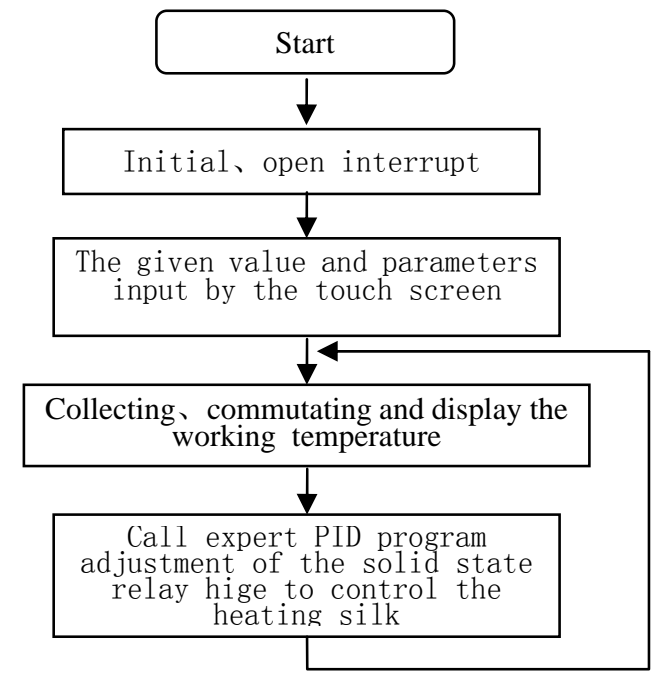

Fig. 4 Main program flowchart

Temperature setting and display system. Temperature setting and display system consists of the MAX485 chip interface, the total MCU (STC89C54RD+) and the touch screen (LJD-eWinA400 (embedded type)) and so on.

MAX485 chip interface. MAX485 interface chip is a RS485 chip of the Maxim company, and it uses $+5 \mathrm{~V}$ single power, $300 \mu \mathrm{A}$ rated current and adopts half-duplex communication. It can convert TTL level to RS-485 level [7].

MAX485 uses multi-point communication standard interface chip which is developed by Electric Industry Association (EIA) of U.S.A, and it uses balanced twisted-pair for transmission line. It uses the balanced drive and differential receiver combination, and common mode interference resistance ability is enhanced, noise interference is better. Maximum transmission distance can achieve $1.2 \mathrm{~km}$. MAX485 can connect up to 32 drives and transceiver with multi-station capability, so that the users can take advantage of a single MAX485 interface to easily establish device network. Receiver sensitivity can reach up to $\pm 200 \mathrm{mV}$, and maximum transmission rate can reach up to $10 \mathrm{Mbps}$. MAX485 has a good application in the long-range, high sensitivity and multi-point communication area. 
Touch screen. The LJD-eWinA400 (embedded) touch screen is used. As a display terminal control equipment, LJD-eWinA400 has a 10.4-inch high-resolution true color digital screen which output resolution is $640 \times 480$, and adopts LED backlight, and it can connect to the lower computer through 232 or 485 interface. This kind of structure which display interface and control system are separated has the advantages that each system can be more independent, without over-reliance on each other, and the system can be convenient to upgrade. LJD-eWinA400 has more perfect man-machine interface system than the traditional display screen. Its transplantation is powerful and it supports .NET Compact Framework2.0 application development, so it simplifies the process of application development on the smart devices and makes the development cycle faster. High level of integration and complete embedded structure make the installation fixed conveniently. And start time is less than 10 seconds, the user memory is up to 60MB.

\section{Summary}

Gasoline-electric hybrid heating not only can improve the thermal efficiency but also can compensate the temperature of the workbench surface that takes advantage of heating oil circulation, especially seal effect of large area module is more obvious. Practice has proved that it avoids temperature gradient and improves the uniformity of temperature that the temperature measurement and control instrument is used to control oil temperature. The precision of temperature control is no more than $0.8^{\circ} \mathrm{C}$, and the non-uniformity is less than $1.2^{\circ} \mathrm{C}$.

\section{References}

[1] Shi Lei, Xia Jinqiang, Zhao Guoyong, etal. "Design of solar cell module laminator based on Pro/E,” Solar Energy, 2008(11): 35-36,in Chinese.

[2] Wei Xing, Wei Hongfou, Wei Hongli. Solar cell module laminator heating platform [P].China:CN201518323U,2010.6.30

[3] Yu Faqing. Transducers Technology and Application[M].China:China Machine Press,2002.

[4] ShiLei, Chen Lidong, Ma Shuying, and etc, "The design and test solar cell module laminator heating system, ” Journal of Hebei Normal University of Science and Technology, vol. 23(4), p.32-35, 2009 (in Chinese).

[5] Wang Jianxin, Ren Yongfeng, Jiao Xinquan. “The Application of Instrument Amplifier AD623 on Data Actuation System,” in Micro-computer Information, vol. 23(1), p.169-180, 2007 (in Chinese).

[6] Li Ping. “Application of solid state relay in solar energy field,” in Solar Energy, 2004(1),p.21-23, in Chinese.

[7] Li Chaoqing. PC and single-chip microcomputer data communication technology [M]. Beijing aerospace university press,2000. 